\title{
Competing forces of nature Improving the odds with full disclosure
}

\author{
Kenneth Maiese ${ }^{1-5}$
}

\begin{abstract}
${ }^{1}$ Division of Cellular and Molecular Cerebral Ischemia; ${ }^{2}$ Departments of Neurology and Anatomy \& Cell Biology; ${ }^{3 B}$ Barbara Ann Karmanos Cancer Institute; ${ }^{4}$ Center for Molecular Medicine and Genetics; ${ }^{5}$ Institute of Environmental Health Sciences; Wayne State University School of Medicine; Detroit, MI USA
\end{abstract}

By their very nature, intrinsic cellular pathways form complex biological systems yet in many ways can open the door for the generation and development of novel therapeutic strategies for many disorders. Interestingly, it is the knowledge gained from examination of these pathways that provide the necessary insight and caution for the application of treatments for different disorders. For example, if one considers the vitamin $\mathrm{B}_{3}$ (niacin) and its amide form nicotinamide, this agent may be seen initially as a necessary nutrient and therapy that is vital for cellular function and deficiency states. Lack of nicotinamide can result in fatigue, loss of appetite, pigmented rashes of the skin and oral ulcerations. More severe states of deficiency lead to pellagra that is characterized by cutaneous rashes, oral ulcerations, gastrointestinal difficulties and cognitive loss. Pellagra can occur during conditions of low nicotinamide or the inability to absorb nicotinamide in the gastrointestinal tract. In addition, excessive alcohol consumption that is associated with poor dietary intake also can lead to severe nicotinamide loss and insufficient absorption. Supplementation with vitamin $\mathrm{B}_{3}$ that can generate nicotinamide through the conversion of nicotinic acid in the liver or through the hydrolysis of $\mathrm{NAD}^{+}$can resolve these disorders.

Interestingly, nicotinamide also is a vital cellular protectant during oxidative stress and cell injury. Administration of nicotinamide during periods of anoxia, oxygen-glucose deprivation or free radical exposure can not only block early cellular apoptotic programs, but also limit inflammatory cell activation. When one translates this work to animal studies, nicotinamide can improve cognitive function, reduce edema following cortical trauma, prevent spinal cord injury and limit disability in models of neurodegeneration and demyelinating disorders.

Yet a paradox ensues upon further consideration of nicotinamide and the pathways controlled by this agent. It appears that a reduction in nicotinamide levels is required to support cellular longevity and increased lifespan. For example, physiological concentrations of nicotinamide can block the activity of the sirtuin Sirt1, a NAD+-dependent deacetylase associated with increased cellular lifespan. In some scenarios, inhibition of sirtuin (Sirt1) activity can be detrimental to cell survival during oxidative stress. As a result, nicotinamide can function as a physiological regulator of sirtuins and supplementation of nicotinamide may at times promote cellular dysfunction or death rather than enhanced cellular survival.

These observations for the agent nicotinamide bring to light the complexity of biological systems and the need for complete understanding of competing pathways. In this issue of Oxidative Medicine and Cellular Longevity, we have selected several insightful papers that provide critical assessment of intrinsic biological pathways during oxidative stress and their ultimate relationship to clinical disease. To begin this process with an excellent overview, Elahi et al. discuss in their paper the varied sources within a cell that can generate reactive oxygen species, how these reactive species can regulate physiological function in the body, and the pathways that can lead to clinical disorders, such as hypertension, atherosclerosis, and cardiac ischemia. Pandey and Rizvi next take us to the world of plant polyphenols and outline the chemical structure and function of these agents, the bioavailability of these agents, and the application of polyphenols to human disease in relation to cancer, diabetes, cardiac disorders, and age-related disorders. In their paper, Maiese et al. focus upon cognitive loss and Alzheimer's disease to elucidate novel treatment strategies with cytokines, such as erythropoietin, and the unique application of forkhead transcription factors. Although these entities can provide new directions for treatment and also are attractive as diagnostic biomarkers, the authors caution on the possible and sometimes unexpected detrimental effects that can ensue and express the necessity for further studies to fully comprehend the cellular pathways controlled by erythropoietin and forkhead transcription factors.

Our research papers in this issue of Oxidative Medicine and Cellular Longevity follow this premise for uncovering the specific mechanisms responsible for the biological activity of a specific agent. In the paper by Tripathi and Chandra that examines the role of plant extracts to treat metabolic disorders, the authors show that the plant extracts of Momordica charantia and Trigonella foenum-graecum have the ability to modulate anti-oxidant pathways as well as control elevated glucose levels in a rat model of diabetes, suggesting a possible role for these derivatives in clinical disease. In a remarkable study also linked to cellular metabolism, Baregamian et al. delve into the pathways that control cellular energy function and mitochondrial autophagy to show that tumor necrosis factor- $\alpha$ and apoptosis signal regulating kinase 1 are primary targets for the control of reactive oxygen species release that lead to cell injury during neonatal necrotizing enterocolitis. Our next four studies highlight additional molecular and signal transduction pathways that can impact the cellular oxidative state during metabolic disorders and nutrition. Rahman et al. investigated the role of the fat-1 transgene that is responsible 
for differential fatty acid changes in the body. They show that fat-1 has broad effects and was associated with limiting pro-inflammatory cytokines, reducing oxidant stress, and promoting the SIRT1 gene that is tied to increased cell longevity. Isoni et al. provide additional insightful knowledge for the etiology of diabetic complications in patients to focus on therapies that can alter cAMP production. They examined peripheral blood mononuclear cells in patients with Type 2 diabetes and performed parallel cell culture studies to show that elevation of cAMP can result in cytokine interleukin-6 production and possibly leading to the pathogenesis of diabetes. Work by Faria et al. extends this work through the examination of intestinal organic cation transport that can significantly affect absorption of both endogenous and exogenous substances. In essence, they show that cellular glutathione levels can alter redox potential and modulate uptake of multiple agents that would in turn determine the course of many disease processes. In our last paper, Baynes and Murray demonstrate that although metabolic disorders such as diabetes may be dependent upon shared cellular pathways, the clinical progression of diabetes may not follow a similar path and progress with independent variables. Such work provides vital implications for the treatment of clinical disease since these authors show in an animal model of diabetes that cardiac and renal disease may proceed independently in diabetes and therefore require strategies that target each separately. For this issue of Oxidative Medicine and Cellular Longevity, our studies serve to illustrate that in regards to oxidative stress and disease progression, the "forces of nature" are always in play and continually require full disclosure with innovative investigations for the design of clinically successful therapies. 


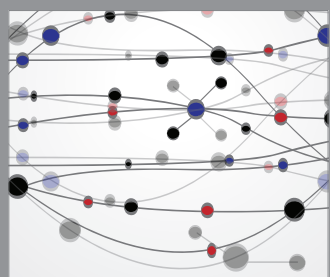

The Scientific World Journal
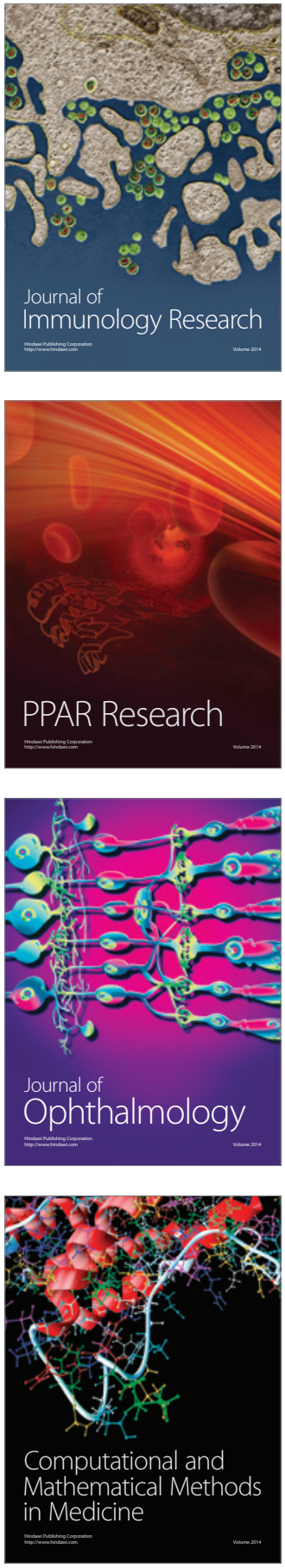

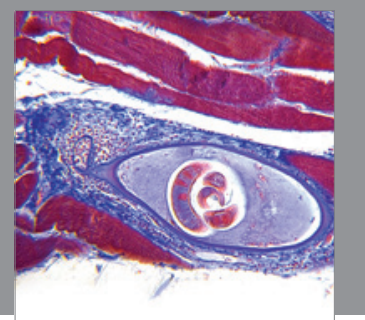

Gastroenterology

Research and Practice
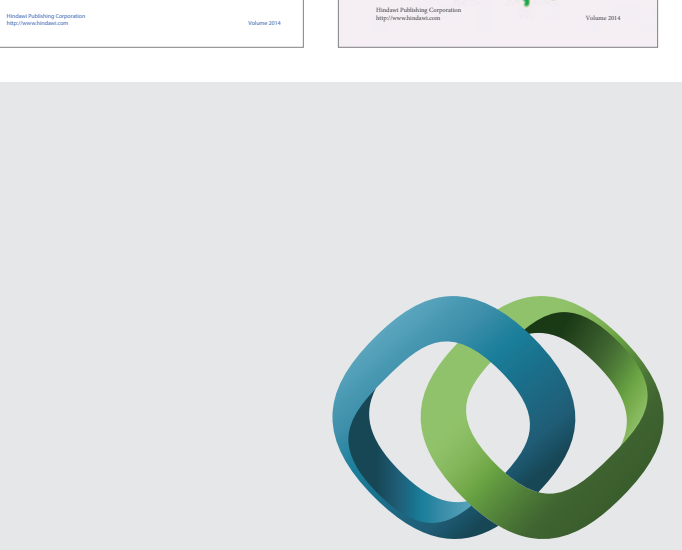

\section{Hindawi}

Submit your manuscripts at

http://www.hindawi.com
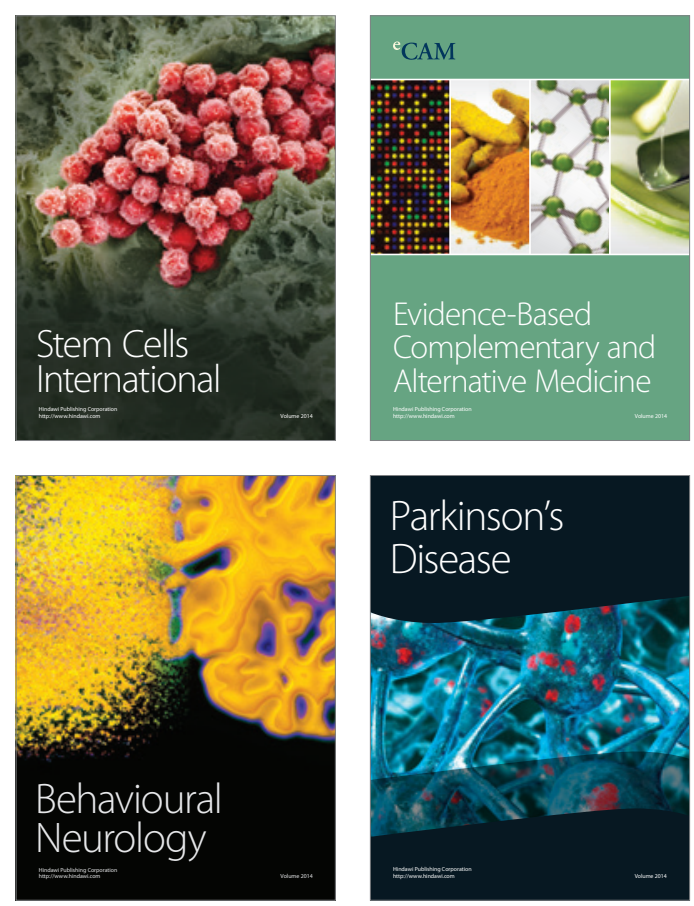

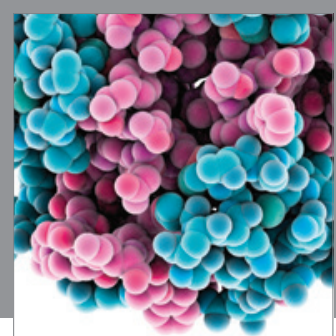

Journal of
Diabetes Research

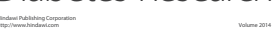

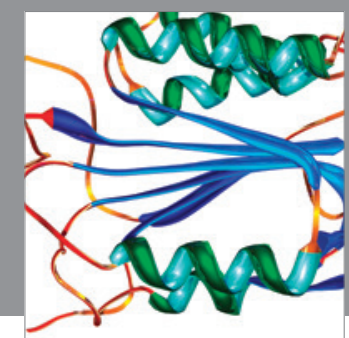

Disease Markers
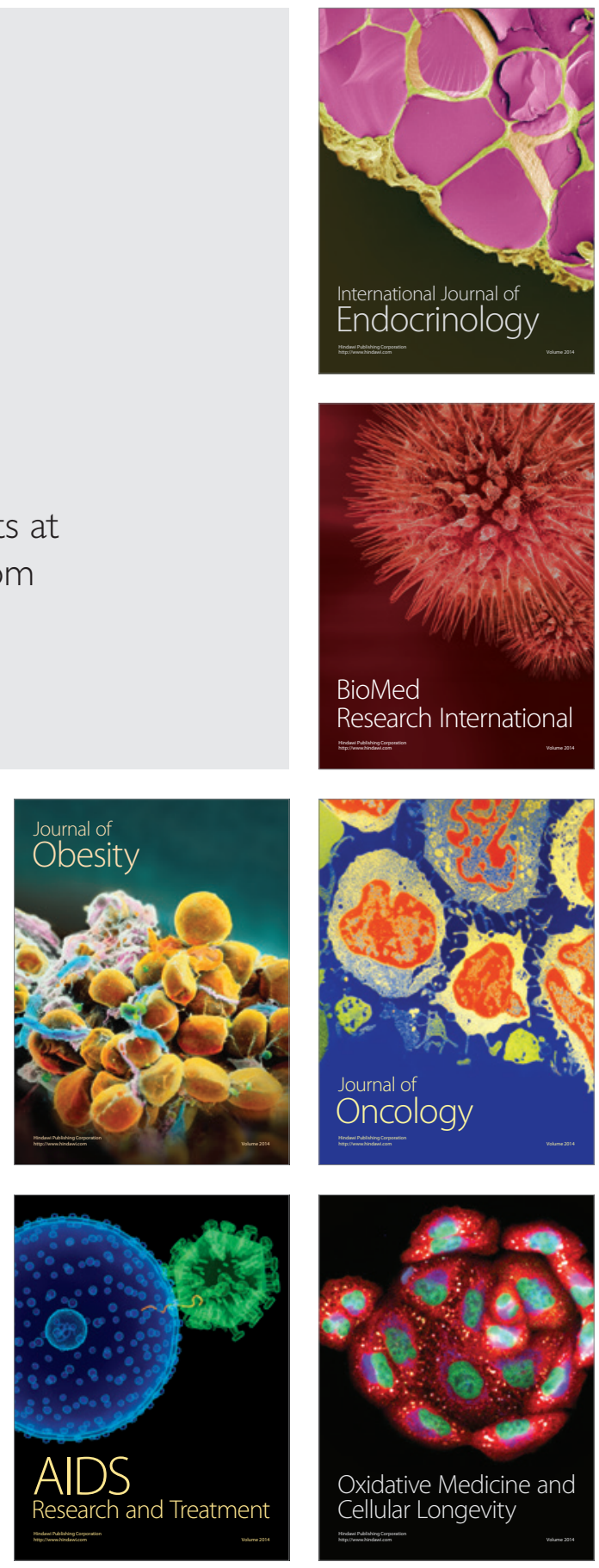